Sie erhoben für die Patienten und die Kontrollen die Häufigkeit von Verurteilungen für Gewaltverbrechen. Zu den Gewaltverbrechen zählten neben Mord und Körperverletzung auch sexuelle Übergriffe, Raub und Nötigung. Dabei muss man wissen, dass in Schweden im Gegensatz zum deutschen Recht auch Menschen als verurteilt gelten, wenn sie nach der Verurteilung in die forensische Psychiatrie eingewiesen werden. Eine Schuldunfähigkeit wegen psychischer Erkrankung gibt es in Schweden also nicht.

In einem Nachbeobachtungszeitraum von etwa 3 Jahren wurden 3,7\% der depressiven Männer und 0,5\% der depressiven Frauen wegen eines Gewaltverbrechens verurteilt. Das ist glücklicherweise nur ein kleiner Anteil der depressiven Patienten. Die Wahrscheinlichkeit, wegen eines Gewaltverbrechens verurteilt zu werden, war in den gematchten Kontrollen jedoch etwa 3-mal geringer (1,2\% bei Männern und $0,2 \%$ bei Frauen). Das entspricht einer Odds Ratio (OR) von 3,0. Besonders häufig waren Gewaltverbrechen bei Männern, die vor der Diagnose einer Depression schon einmal wegen eines derartigen Verbrechens verurteilt waren. Von ihnen wurde im Nachbeobachtungszeitraum jeder 8. wegen eines Gewaltverbrechens verurteilt (12,5\%). Auch andere Faktoren wie das Bestehen von substanzbezogenen Störungen und selbstschädigendem Verhalten in der Vergangenheit erhöhten die Wahrscheinlichkeit, wegen eines Gewaltverbrechens verurteilt zu werden. Selbst Patienten, die keinen dieser 3 Risikofaktoren hatten, wurden häufiger als die gesunden Kontrollen wegen Gewaltverbrechen verurteilt (2,2\% der Männer und 0,3 $\%$ der Frauen).

Die beobachtete Häufigkeit von Gewaltverbrechen ist glücklicherweise gering. Sie ist beispielsweise geringer als die Häufigkeit bei schizophrenen Patienten in einem vergleichbaren Beobachtungszeitraum (10\% der Männer). Die Rate ist jedoch vergleichbar mit der Häufigkeit von selbstschädigendem Verhalten bei depressiven Männern in dieser Studie
(3,3\%). Bei depressiven Frauen in dieser Studie war die Häufigkeit von selbstschädigendem Verhalten (4,3\%) jedoch deutlich höher als die Häufigkeit von Verurteilungen wegen Gewaltverbrechen (0,5\%). Wegen der geringen Häufigkeit von selbstverletzendem Verhalten und Tod durch Suizid bei den Kontrollen ist hier die OR besonders hoch (5,7 bzw. 6,7).

Fazit Glücklicherweise kommen Gewaltverbrechen bei depressiven Patienten nur selten vor. Dennoch: Insbesondere bei depressiven Männern sollte neben dem Risiko für Selbstschädigungen immer auch das Risiko für fremdschädigendes Verhalten erhoben werden. Denn beides kommt bei Männern in etwa gleich häufig vor.

Dr. Jan Philipp Klein, Lübeck

\section{Wirken Psychopharmaka karzinogen?}

Amerio A et al. Carcinogenicity of psychotropic drugs: A systematic review of US Food and Drug Administration - required preclinical in vivo studies. Aust N Z J Psychiatry 2015; DOI: 10.1177/000486741 5582231

Der Gebrauch von Psychopharmaka ist in den letzten Jahren dramatisch gestiegen. In den USA stieg die Verschreibung dieser Medikamentengruppe von 1996-2005 um $74 \%$, wovon die meisten Medikamente von den Hausärzten verschrieben werden. Vor dem Hintergrund des massiven Gebrauchs dieser Medikamente sollte auch das karzinogene Risiko berücksichtigt werden. Für die Zulassung neuer Medikamente fordert die amerikanische $\mathrm{Zu}$ lassungsbehörde (FDA) eine Serie von Studien, welche die Sicherheit der neuen Medikamente nachweisen. In diesem Zusammenhang wurden auch schon Artikel veröffentlicht, die eine gewisse Besorgnis in Bezug auf eine erhöhte Karzinogenität postuliert haben. Allerdings sind klinische Studien häufig sehr heterogen, sodass eine Vergleichbarkeit nicht immer möglich ist.
Dies haben die Autoren der aktuellen Studie zum Anlass genommen, die von der FDA gestützten präklinischen In-vivo-Studien in Bezug auf ihre Karzinogenität zu vergleichen. Hierzu wurden auch unveröffentlichte Daten der FDA abgefragt. Die Autoren bildeten folgende Medikamentengruppen: Antidepressive, Antipsychotika, Bezodiazepine/Sedativa, Amphetamine und Stimulanzien, Lithium und Antikonvulsiva. 71,4\% (30/42) aller untersuchten Pharmaka zeigten eine präklinische Evidenz für eine erhöhte Karzinogenität. Bei den Antidepressiva zeigten 63,6\% eine Assoziation, wobei diese nicht spezifisch für Wirkstoffgruppen war. So zeigte sich z. B. eine Assoziation bei Sertralin und Citalopram, nicht aber bei Fluoxetin. Bei den antipsychotisch wirksamen Medikamenten zeigten $90 \%$ eine Assoziation. Hier konnte lediglich bei Clozapin kein Hinweis gefunden werden. Bei den Benzodiazepinen und Sedativa gab es bei $70 \%$ und bei den Antikonvulsiva bei 85,7\% der Medikamente eine Assoziation. Bei den Stimulanzien konnte dies lediglich für Methylphenidat gezeigt werden. Dies entsprach einem Prozentsatz von 25\%.

Einschränkend muss natürlich erwähnt werden, dass die präklinischen Studien an Mäusen und Ratten durchgeführt worden sind, die z.T. per se eine erhöhte Inzidenz für spezifische Tumoren zeigen. Zudem werden in diesen Studien sehr hohe Medikamentendosen verwendet, um einen möglichen Effekt detektieren zu können. Des Weiteren ist es nicht möglich von einem Tiermodell direkte Rückschlüsse auf den Menschen zu ziehen. Hier wurden auch schon gegenteilige Studien veröffentlich, die eine reduzierte Karzinogenität festgestellt haben und diese mit verbesserten immunologischen Effekten begründeten.

Fazit Aus präklinischen In-vivo-Tiermodellen können zwar keine abschließenden Schlussfolgerungen für den Menschen gezogen werden. Nichtsdestotrotz plädieren die Autoren dafür diese Ergebnisse der Grundlagenforschung nicht zu ignorieren.

Dr. Bastian Willenborg, Wendisch-Rietz 